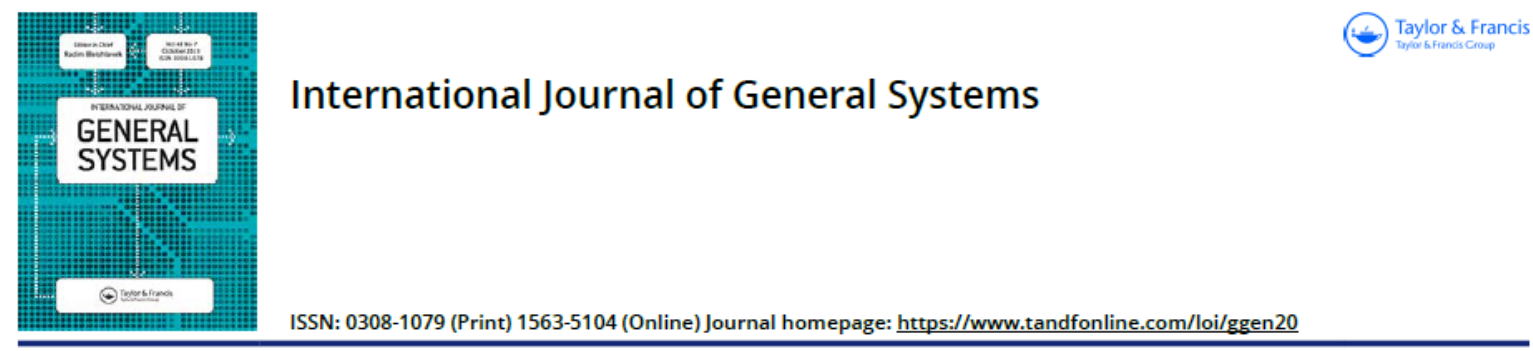

\title{
Centralized, distributed and sequential fusion estimation from uncertain outputs with correlation between sensor noises and signal
}

R. Caballero-Águila, A. Hermoso-Carazo \& J. Linares-Pérez

\section{Preprint version. Please cite original version:}

R. Caballero-Águila, A. Hermoso-Carazo \& J. Linares-Pérez (2019) Centralized, distributed and sequential fusion estimation from uncertain outputs with correlation between sensor noises and signal, International Journal of General Systems, 48 (7), 713-737

\section{https://doi.org/10.1080/03081079.2019.1659257}

\begin{abstract}
This paper focuses on the least-squares linear fusion filter design for discrete-time stochastic signals from multisensor measurements perturbed not only by additive noise, but also by different uncertainties that can be comprehensively modeled by random parameter matrices. The additive noises from the different sensors are assumed to be cross-correlated at the same time step and correlated with the signal at the same and subsequent time steps. A covariance based approach is used to derive easily implementable recursive filtering algorithms under the centralized, distributed and sequential fusion architectures. Although centralized and sequential estimators both have the same accuracy, the evaluation of their computational complexity reveals that the sequential filter can provide a significant reduction of computational cost over the centralized one. The accuracy of the proposed fusion filters is explored by a simulation example, where observation matrices with random parameters are used to describe different kinds of sensor uncertainties.
\end{abstract}




\section{Introduction}

Multisensor systems are applied with different purposes in a large variety of fields in engineering, technology or computer science, among others. These applications demand an efficient use of the information contained in all the sensor observations that must be used to estimate the signal of interest and, for that purpose, the use of suitable information fusion techniques is required. If a central processor directly receives all the measured outputs from sensors and processes them in real time, the corresponding estimator is known as centralized fusion estimator (Ma and Sun (2013), CaballeroÁguila et al. (2015)). Since the centralized approach uses all sensor observations, it clearly provides optimal estimators, but it suffers from several drawbacks, such as poor reliability, heavy communication and heavy computational burden.

A different approach is the commonly named distributed or decentralized fusion method (Dong et al. (2015), Caballero-Águila et al. (2017)). Under this approach, each single sensor produces its own local estimator based only on its own data. These local estimators are then transmitted to a central processor where they are fused to yield an estimator that outperforms the local ones in terms of some optimality criterion (e.g. the least-squares criterion). For a detailed overview of recent advances on distributed filtering for stochastic systems over sensor networks, the reader may go through references Ding et al. (2014) and Sun et al. (2017a). Even though the distributed fusion architecture is more robust and flexible, has better reliability and usually lower computational load, it provides less accurate estimators in general. Both centralized and distributed fusion methods are based on the idea of batch fusion, meaning that sensor measurements or local estimates are fused all at a time when all of them are available at the fusion center.

The sequential fusion method, where the sensor data are fused one at a time, according to the arrival time order (instead of processing them as a whole vector), overcomes the aforementioned issues, achieving the same estimation accuracy but a lower computational cost than the centralized one. For this reason, the sequential fusion estimation problem in multisensor systems has attracted much research interest during the last decade and, assuming the knowledge of the state-space model of the system, many relevant results have been presented in Wen et al. (2013), Yan et al. (2013), Zhou et al. (2016), Kettner and Paolone (2017), Lin and Sun (2018), Feng et al. (2018) and Lin and Sun (2019), among others. In Yan et al. (2013), the sequential fusion estimation for a class of discrete time-varying linear systems is addressed, when the noises of different sensors are cross-correlated and also coupled with the system noise of the previous step. Since the estimators of the measurement noises are ignored, the sequential fusion algorithm proposed by these authors provides only suboptimal estimators. More recently, a linear optimal recursive sequential fusion filtering algorithm for multisensor systems with stochastic parameter perturbations and fading measurements has been proposed in Lin and Sun (2018), under the additional assumption that the measurement noises of different sensors are correlated with each other and also correlated with the system noise at the same time step. In Lin and Sun (2019), the optimal sequential estimation problem is studied for multisensor systems with correlated noises, where the measurement noises of the different sensors are cross-correlated with each other at the same instant and correlated with the system noise at the previous step.

In some conventional algorithms, additive noise is assumed to be the only source of uncertainty in the sensor measurements and perfect transmissions between the sensor 
nodes and the fusion center are considered (see e.g., Wen et al. (2013), Yan et al. (2013) and references therein). However, in many practical applications, a more realistic scenario had better include the presence of different random phenomena (stochastic parameter perturbations, missing or fading measurements, multiplicative noise, etc.) in the sensor output measurements, as such uncertainties are usually inherent to the network itself, due to bandwidth limitations or measuring device inaccuracies (see Liu et al. (2016a), Liu et al. (2016b), Hu et al. (2017), Li et al. (2017), Wang and Sun (2017) and Zhang et al. (2018), among others). To cope with these kind of situations, a proper choice is the inclusion of random parameter matrices in the measurement equation, as they provide an inclusive framework to model many of those random phenomena. For this reason, the estimation problem in systems with random parameter matrices has sparked great research interest over the last years (see Yang et al. (2016), Sun et al. (2017b), Wang and Zhou (2017), Caballero-Águila et al. (2018), Caballero-Águila et al. (2019b) and references therein).

Another prolific research topic arises when dealing with multisensor systems whose noises at different sensors are cross-correlated and also coupled with the process noise. Sensor noise cross-correlation can be found when the signal process is observed by sensors that operate in a common noisy environment (Caballero-Águila et al. (2017), Li et al. (2017), Sun et al. (2017b), Wang and Sun (2017)), but it also can arise, for example, after the transformation of systems with random delays or packet-dropouts (Zhu et al. (2013)). Concerning the correlation between the sensor measurement noises and the system noise, although several papers consider the estimation problem when the sensor noises are correlated with the system noise at the same time step (Wen et al. (2013), Lin and Sun (2018)), the case of correlation in the previous time step is also of great interest (Yan et al. (2013), Lin and Sun (2019)). One of the most common situations in which this type of correlation stems is the procedure of discretization of continuous-time systems; actually, if the discrete-time linear system is obtained from discretization of a continuous-time system, then the measurement noise is correlated with the system noise of the previous time step (Li (2003)). This correlation assumption makes the measurement noises at any time step be correlated with the signal at the same and subsequent time steps.

Finally, it should be pointed out that, to the best of the authors' knowledge, all papers in the literature on sequential fusion estimation require the knowledge of the state-space model; hence, the use of covariance information instead is a challenging research field. It is also important to mention that all papers on covariance-based centralized and distributed fusion algorithms assume independence between the signal and sensor noises and, hence, independence between the system noise and sensor noises is required, which, as already indicated, is not a realistic assumption in many practical situations.

Encouraged by the above considerations, our goal is to design fusion algorithms, based on the use of covariance information, for the least-squares linear centralized, distributed and sequential filtering estimators of a stochastic signal, measured by a multisensor network, when the additive noises are correlated across the different sensors and also correlated with the signal process.

Compared with the related work in the literature, the main contributions are:

(a) The existing results on the sequential fusion estimation problem mainly focus on the state-space model. In this paper, however, the signal evolution model 
is assumed to be unknown and only the mean and covariance functions of the processes involved in the sensor measurement equations are available; actually, this is the first time the sequential fusion estimation problem have been addressed in this covariance information context.

(b) Unlike previous authors' work concerning centralized and distributed fusion estimation algorithms, where independence between the signal and the additive noises of the different sensors has always been assumed, the fusion estimation algorithms proposed in this paper are obtained under the hypothesis that the additive sensor noises are correlated with the signal at the same and subsequent time steps.

The rest of the paper is organized as follows. In Section 2, the observation model and the hypotheses about the stochastic processes involved in such model are described. The centralized and distributed fusion filtering algorithms are detailed in Section 3 and Section 4, respectively. The sequential fusion filtering algorithm is derived in Section 5. In Section 6, the feasibility and effectiveness of the proposed fusion filters is verified by a computer simulation example, where the influence of the sensor uncertainties on the performance of the filters is analyzed. Also, this example shows the equivalence on estimation accuracy of the sequential and centralized fusion filters, and their superiority over the distributed one. Finally, some concluding remarks are drawn in Section 7 and the derivation of the centralized, distributed and sequential estimation algorithms are detailed in appendices A, B and C, respectively.

Notation: The $n$-dimensional Euclidean space and the set of all $n_{1} \times n_{2}$ real matrices are denoted by $\mathbb{R}^{n}$ and $\mathbb{R}^{n_{1} \times n_{2}}$, respectively. If $A$ and $B$ are equal by definition (i.e., $A$ is defined as $B$ ), it will be symbolically written as $A \equiv B$. For a matrix $M, M^{T}$ and $M^{-1}$ denote its transpose and inverse, respectively; also for a vector $w$, its transpose is denoted by $w^{T}$. If the dimensions of a vector or a matrix are not explicitly stated, they are assumed to be compatible with algebraic operations. $I$ and 0 denote the identity matrix and the zero matrix, respectively. The shorthand $\left(M_{1}, \ldots, M_{s}\right)$ denotes a partitioned matrix whose blocks are the submatrices $M_{1}, \ldots, M_{s}$. For any function $G_{k, h}$, depending on the time instants $k$ and $h$, we will write $G_{k}=G_{k, k}$ for simplicity; analogously, $F^{(i)}=F^{(i i)}$ will be written for any function $F^{(i j)}$, depending on the sensors $i$ and $j$. Finally, $\delta_{k, h}$ denotes the Kronecker delta function, which is equal to one if $k=h$ and zero otherwise.

\section{Observation model. Assumptions}

This paper addresses the least-squares (LS) linear fusion estimation problem of discrete-time random signals from multi-sensor noisy measurements, perturbed by random parameter matrices, using different fusion methods; specifically, the centralized, distributed and sequential methods are considered. The estimation problem will be addressed by an innovation approach, since the LS linear signal estimator based on the observations agrees with the one based on the innovations (Kailath et al. (2000)). The fusion estimation algorithms will be derived using only covariance information, without requiring full knowledge of the evolution model generating the signal process. 


\subsection{Signal processes with factorizable covariance function}

The estimation algorithms based on the state-space model require an explicit mathematical model of the signal time-variation and an explicit model expressing how the observations used to estimate the signal are related with it. Hence, for every signal evolution model, a different estimation algorithm must be derived. This handicap can be overcome using a covariance-based approach for the algorithm design, where the evolution model of the signal process is not required but, instead, it must be assumed that the signal mean function is zero and its covariance function is factorizable. More precisely, the following assumption is required:

(A1) The $n_{x}$-dimensional signal $\left\{x_{k}\right\}_{k \geq 1}$ is a zero-mean second-order process and its autocovariance function is expressed in a separable form; namely, $E\left[x_{k} x_{h}^{T}\right]=$ $A_{k} B_{h}^{T}, h \leq k$, where $A_{k}, B_{h} \in \mathbb{R}^{n_{x} \times n}$ are known matrices.

It should be pointed out that this hypothesis holds true for the signal process of the most commonly used signal evolution models and, consequently, the covariancebased estimation approach provides a comprehensive context to cope with different signal models without the necessity of obtaining a specific algorithm for each of them. Some examples of processes satisfying (A1) are the signal of linear systems or that of uncertain systems with state-dependent multiplicative noise (for details see e.g. Caballero-Águila et al. (2019a)). Also, signals involving a sum of multiple multiplicative noise terms, as those considered in Lin and Sun (2018), meet this assumption, as it will be shown in Section 6 .

\subsection{Multi-sensor measured outputs with random parameter matrices}

Consider the problem of estimating the signal $x_{k}$ from a set of measurements that are provided by $m$ sensors and obey the following model:

$$
y_{k}^{(i)}=H_{k}^{(i)} x_{k}+v_{k}^{(i)}, \quad k \geq 1, \quad i=1, \ldots, m,
$$

where $y_{k}^{(i)} \in \mathbb{R}^{n_{y}}$ is the measured output of the $i t h$ sensor at time $k, H_{k}^{(i)} \in \mathbb{R}^{n_{y} \times n_{x}}$, and $v_{k}^{(i)} \in \mathbb{R}^{n_{y}}$ is the measurement noise vector.

On the sensor noise correlation. The additive noises of the different sensors are assumed to be cross-correlated at the same time and also correlated with the signal process; specifically, the following two assumptions are required:

(A2) The measurement noises $\left\{v_{k}^{(i)}\right\}_{k \geq 1}, i=1, \ldots, m$, are zero-mean white secondorder process with known $E\left[v_{k}^{(i)} v_{h}^{(j) T}\right]=R_{k}^{(i j)} \delta_{k, h}, i, j=1, \ldots, m$.

(A3) For $i=1, \ldots, m$, the noise $\left\{v_{k}^{(i)}\right\}_{k \geq 1}$ is correlated with the signal process $\left\{x_{k}\right\}_{k \geq 1}$ according to the following correlation function:

$$
E\left[x_{k} v_{h}^{(i) T}\right]= \begin{cases}C_{k} D_{h}^{(i) T}, & h \leq k \\ 0, & h>k\end{cases}
$$

where $C_{k} \in \mathbb{R}^{n_{x} \times n^{\prime}}$ and $D_{h}^{(i)} \in \mathbb{R}^{n_{y} \times n^{\prime}}$ are known matrices.

These two assumptions allow us to consider state-space models in which the additive 
noises of different sensors are correlated with each other at the same time instant, and also correlated with the system noise of the previous time step (see Section 6). This correlation assumption between the measurement noises and the system noise appears, for example, in Lin and Sun (2019) and also when the discrete-time linear system is obtained from discretization of a continuous-time system (Yan et al. (2013)).

On the sensor measurement matrices. In different real situations, the unreliable environment or fallible operating mechanisms may yield sensor failures that cannot be described using only additive noises; for instance, uncertainties such as missing or fading measurements, sensor gain degradation, or presence of multiplicative noises. Hence, besides the noises $v_{k}^{(i)}$, the measured outputs $y_{k}^{(i)}$ are here considered subject to other stochastic uncertainties, coming from multiple sources, which can be mathematically described in a unified way by assuming that the parameter matrices $H_{k}^{(i)}$ in (1) are random. In order that the random measurement matrices include the aforementioned uncertainties as special cases, no specific distribution for their elements will be required, and only the existence and knowledge of their first and second-order moments will be assumed. Moreover, to cover the possibility of having the same uncertainty in the different sensors, we will suppose that the elements of the matrices modelling these uncertainties in the different sensors are cross-correlated in the same time instant. More specifically, the following assumption on the measurement matrices in (1) is considered:

(A4) For $i=1, \ldots, m,\left\{H_{k}^{(i)}\right\}_{k \geq 1}$ are sequences of independent random parameter matrices. By denoting $h_{k, p q}^{(i)}, p=1, \ldots, n_{y}$ and $q=1, \ldots, n_{x}$, the $(p, q)$ th entry of $H_{k}^{(i)}$, it is assumed that the means, $E\left[h_{k, p q}^{(i)}\right]$, and the covariances, $\operatorname{Cov}\left[h_{k, p q}^{(i)}, h_{k^{\prime} p^{\prime} q^{\prime}}^{(j)}\right]=\operatorname{Cov}\left[h_{k, p q}^{(i)}, h_{k, p^{\prime} q^{\prime}}^{(j)}\right] \delta_{k, k^{\prime}}$, for $p, p^{\prime}=1, \ldots, n_{y}$ and $q, q^{\prime}=$ $1, \ldots, n_{x}$, are known.

This assumption provides an inclusive framework to model different sensor uncertainties, for instance, the fading measurement phenomenon considered in Lin and Sun (2018).

Also, the following independence assumption is assumed:

(A5) $\left(\left\{x_{k}\right\}_{k \geq 1},\left\{v_{k}^{(i)}\right\}_{k \geq 1}, i=1, \ldots, m\right)$ and $\left(\left\{H_{k}^{(i)}\right\}_{k \geq 1}, i=1, \ldots, m\right)$ are independent.

Properties. Taking into account the above assumptions (A1)-(A5), the following properties, which will be used in the derivation of the distributed and sequential fusion algorithms, are easily derived:

- Denoting $\bar{H}_{k}^{(i)} \equiv E\left[H_{k}^{(i)}\right]$ and $\widetilde{H}_{k}^{(i)} \equiv H_{k}^{(i)}-\bar{H}_{k}^{(i)}$, from (A1) and the conditional expectation properties, we have

$$
E\left[\widetilde{H}_{k}^{(i)} x_{k} x_{k}^{T} \widetilde{H}_{k}^{(j) T}\right]=E\left[\widetilde{H}_{k}^{(i)} A_{k} B_{k}^{T} \widetilde{H}_{k}^{(j) T}\right], \quad i, j=1, \ldots, m,
$$


where, for $p, q=1, \ldots, n_{y}$, the $(p, q)$-th entries of these matrices are

$$
\left(E\left[\widetilde{H}_{k}^{(i)} A_{k} B_{k}^{T} \widetilde{H}_{k}^{(j) T}\right]\right)_{p q}=\sum_{a=1}^{n_{x}} \sum_{b=1}^{n_{x}} \operatorname{Cov}\left[h_{k, p a}^{(i)}, h_{k, q b}^{(j)}\right]\left(A_{k} B_{k}^{T}\right)_{a b} .
$$

- The signal process, $\left\{x_{k}\right\}_{k \geq 1}$, is correlated with the measurement process, $\left\{y_{k}^{(i)}\right\}_{k \geq 1}$, and

$$
E\left[x_{k} y_{h}^{(i) T}\right]=\left(A_{k}, C_{k}\right)\left(\bar{H}_{h}^{(i)} B_{h}, D_{h}^{(i)}\right)^{T}, \quad h \leq k, \quad i=1, \ldots, m
$$

- The random vectors $\widetilde{H}_{k}^{(i)} x_{k}+v_{k}^{(i)}$ and $y_{k}^{(j)}$ are correlated, for all $i, j=1, \ldots, m$, with

$$
E\left[\left(\widetilde{H}_{k}^{(i)} x_{k}+v_{k}^{(i)}\right) y_{k}^{(j) T}\right]=E\left[\widetilde{H}_{k}^{(i)} A_{k} B_{k}^{T} \widetilde{H}_{k}^{(j) T}\right]+D_{k}^{(i)} C_{k}^{T} \bar{H}_{k}^{(j) T}+R_{k}^{(i j)} .
$$

\section{Centralized fusion filtering estimators}

In the centralized fusion method, all the measurement data coming from the different sensors are sent to a fusion center where they are combined to provide optimal estimators based on all the received measurements. So, to address the LS estimation problem by this method, in which the observations from the different sensors are jointly processed at each sampling time, the observation model (1) is rewritten in a stacked form as shown in the next subsection.

\subsection{Stacked observation model. Properties}

Defining the vectors $y_{k}=\left(y_{k}^{(1) T}, \ldots, y_{k}^{(m) T}\right)^{T}$, the observation equations of the different sensors (1) are rewritten in a compact way as follows:

$$
y_{k}=H_{k} x_{k}+v_{k}, \quad k \geq 1,
$$

where $H_{k}=\left(H_{k}^{(1) T}, \ldots, H_{k}^{(m) T}\right)^{T}$ and $v_{k}=\left(v_{k}^{(1) T}, \ldots, v_{k}^{(m) T}\right)^{T}$.

To address the LS estimation problem from the observations given in (4), the following statistical properties of the processes involved in (4), which are easily inferred from assumptions (A2)-(A5), are used:

- $\left\{H_{k}\right\}_{k \geq 1}$ is a sequence of independent random matrices with known means, $\bar{H}_{k} \equiv$ $E\left[H_{k}\right]=\left(\bar{H}_{k}^{(1) T}, \ldots, \bar{H}_{k}^{(m) T}\right)^{T}$, and

$$
E\left[\widetilde{H}_{k} x_{k} x_{k}^{T} \widetilde{H}_{k}^{T}\right]=E\left[\widetilde{H}_{k} A_{k} B_{k}^{T} \widetilde{H}_{k}^{T}\right]=\left(E\left[\widetilde{H}_{k}^{(i)} A_{k} B_{k}^{T} \widetilde{H}_{k}^{(j) T}\right]\right)_{i, j=1, \ldots, m} .
$$

- The noise process, $\left\{v_{k}\right\}_{k \geq 1}$, has zero mean and $E\left[v_{k} v_{h}^{T}\right]=R_{k} \delta_{k, h}$, where $R_{k}=$ $\left(R_{k}^{(i j)}\right)_{i, j=1, \ldots, m}$.

- The signal process, $\left\{x_{k}\right\}_{k \geq 1}$, and the noise process, $\left\{v_{k}\right\}_{k \geq 1}$, are correlated to each other, with $E\left[x_{k} v_{h}^{T}\right]=\left\{\begin{array}{ll}C_{k} D_{h}^{T}, & h \leq k, \\ 0, & h>k .\end{array}\right.$ where $D_{h}=\left(D_{h}^{(1) T}, \ldots, D_{h}^{(m) T}\right)^{T}$. 
- $\left\{H_{k}\right\}_{k \geq 1}$ is independent of $\left(\left\{x_{k}\right\}_{k \geq 1},\left\{v_{k}\right\}_{k \geq 1}\right)$.

- The signal process, $\left\{x_{k}\right\}_{k \geq 1}$, is correlated with the process $\left\{y_{k}\right\}_{k \geq 1}$ and

$$
E\left[x_{k} y_{h}^{T}\right]=\left(A_{k}, C_{k}\right)\left(\bar{H}_{h} B_{h}, D_{h}\right)^{T}, \quad h \leq k .
$$

- The random vectors $\widetilde{H}_{k} x_{k}+v_{k}$ and $y_{k}$ are correlated to each other, with

$$
E\left[\left(\widetilde{H}_{k} x_{k}+v_{k}\right) y_{k}^{T}\right]=E\left[\widetilde{H}_{k} A_{k} B_{k}^{T} \widetilde{H}_{k}^{T}\right]+D_{k} C_{k}^{T} \bar{H}_{k}^{T}+R_{k}, \quad k \geq 1 .
$$

\subsection{Centralized filtering recursive algorithm}

A recursive algorithm for the LS linear centralized fusion filter, $\widehat{x}_{k / k}^{(C)}$, of the signal $x_{k}$ based on the observations $\left\{y_{1}, \ldots, y_{k}\right\}$ given in (4), is presented in the following theorem.

Theorem 3.1. Under assumptions (A1)-(A5), the centralized filter, $\widehat{x}_{k / k}^{(C)}$, and the filtering error covariance matrix, $\Sigma_{k / k}^{(C)} \equiv E\left[\left(x_{k}-\widehat{x}_{k / k}^{(C)}\right)\left(x_{k}-\widehat{x}_{k / k}^{(C)}\right)^{T}\right]$, are given by

$$
\begin{gathered}
\widehat{x}_{k / k}^{(C)}=\left(A_{k}, C_{k}\right) u_{k}, \quad k \geq 1, \\
\Sigma_{k / k}^{(C)}=A_{k} B_{k}^{T}-\left(A_{k}, C_{k}\right) K_{k}^{u}\left(A_{k}, C_{k}\right)^{T}, \quad k \geq 1,
\end{gathered}
$$

where the vectors $u_{k}$ and the matrices $K_{k}^{u} \equiv E\left[u_{k} u_{k}^{T}\right]$ are obtained from

$$
\begin{gathered}
u_{k}=u_{k-1}+\mathcal{U}_{k} \Pi_{k}^{-1} \mu_{k}, \quad k \geq 1 ; \quad u_{0}=0, \\
K_{k}^{u}=K_{k-1}^{u}+\mathcal{U}_{k} \Pi_{k}^{-1} \mathcal{U}_{k}^{T}, \quad k \geq 1 ; \quad K_{0}^{u}=0,
\end{gathered}
$$

and the matrices $\mathcal{U}_{k} \equiv E\left[u_{k} \mu_{k}^{T}\right]$ satisfy

$$
\mathcal{U}_{k}=\left(\bar{H}_{k} B_{k}, D_{k}\right)^{T}-K_{k-1}^{u}\left(A_{k}, C_{k}\right)^{T} \bar{H}_{k}^{T}, \quad k \geq 1 .
$$

The innovation, $\mu_{k} \equiv y_{k}-\widehat{y}_{k / k-1}^{(C)}$, and its covariance matrix, $\Pi_{k} \equiv E\left[\mu_{k} \mu_{k}^{T}\right]$, are given by

$$
\begin{gathered}
\mu_{k}=y_{k}-\bar{H}_{k}\left(A_{k}, C_{k}\right) u_{k-1}, \quad k \geq 1, \\
\Pi_{k}=E\left[\widetilde{H}_{k} A_{k} B_{k}^{T} \widetilde{H}_{k}^{T}\right]+D_{k} C_{k}^{T} \bar{H}_{k}^{T}+R_{k}+\bar{H}_{k}\left(A_{k}, C_{k}\right) \mathcal{U}_{k}, \quad k \geq 1 .
\end{gathered}
$$

Proof. See Appendix A.

\section{Distributed fusion estimation problem}

Under the distributed fusion methodology, local filtering estimators are computed for each single sensor $i=1, \ldots, m$, using only the measurements of the sensor itself; after that, all the local filters are sent to a fusion center to generate the estimator by a 
certain fusion criterion. Accordingly, in this section, we will firstly obtain a recursive algorithm for the local LS linear filtering estimators (Theorem 4.1) and, after that, the proposed distributed filtering estimators will be designed as the LS matrix-weighted linear combination of the local filters (Theorem 4.2).

Theorem 4.1. Under assumptions (A1)-(A5), for each $i=1, \ldots, m$, the local $L S$ linear filter, $\widehat{x}_{k / k}^{(i)}$, and the corresponding error covariance matrix, $\Sigma_{k / k}^{(i)} \equiv E\left[\left(x_{k}-\right.\right.$ $\left.\left.\widehat{x}_{k / k}^{(i)}\right)\left(x_{k}-\widehat{x}_{k / k}^{(i)}\right)^{T}\right]$, are given by

$$
\begin{gathered}
\widehat{x}_{k / k}^{(i)}=\left(A_{k}, C_{k}\right) u_{k}^{(i)}, \quad k \geq 1, \\
\Sigma_{k / k}^{(i)}=A_{k} B_{k}^{T}-\left(A_{k}, C_{k}\right) K_{k}^{u^{(i)}}\left(A_{k}, C_{k}\right)^{T}, \quad k \geq 1,
\end{gathered}
$$

where the vectors $u_{k}^{(i)}$ and the matrices $K_{k}^{u^{(i)}} \equiv E\left[u_{k}^{(i)} u_{k}^{(i) T}\right]$ are obtained from

$$
\begin{gathered}
u_{k}^{(i)}=u_{k-1}^{(i)}+\mathcal{U}_{k}^{(i)} \Pi_{k}^{(i)-1} \mu_{k}^{(i)}, \quad k \geq 1 ; \quad u_{0}^{(i)}=0, \\
K_{k}^{u^{(i)}}=K_{k-1}^{u^{(i)}}+\mathcal{U}_{k}^{(i)} \Pi_{k}^{(i)-1} \mathcal{U}_{k}^{(i) T}, \quad k \geq 1 ; \quad K_{0}^{u^{(i)}}=0,
\end{gathered}
$$

and the matrices $\mathcal{U}_{k}^{(i)} \equiv E\left[u_{k}^{(i)} \mu_{k}^{(i) T}\right]$ satisfy

$$
\mathcal{U}_{k}^{(i)}=\left(\bar{H}_{k}^{(i)} B_{k}, D_{k}^{(i)}\right)^{T}-K_{k-1}^{u^{(i)}}\left(A_{k}, C_{k}\right)^{T} \bar{H}_{k}^{(i) T}, \quad k \geq 1 .
$$

The innovations, $\mu_{k}^{(i)} \equiv y_{k}^{(i)}-\widehat{y}_{k / k-1}^{(i)}$, and their covariance matrices, $\Pi_{k}^{(i)} \equiv$ $E\left[\mu_{k}^{(i)} \mu_{k}^{(i) T}\right]$, are given by

$$
\begin{gathered}
\mu_{k}^{(i)}=y_{k}^{(i)}-\bar{H}_{k}^{(i)}\left(A_{k}, C_{k}\right) u_{k-1}^{(i)}, \quad k \geq 1, \\
\Pi_{k}^{(i)}=E\left[\widetilde{H}_{k}^{(i)} A_{k} B_{k}^{T} \widetilde{H}_{k}^{(i) T}\right]+D_{k}^{(i)} C_{k}^{T} \bar{H}_{k}^{(i) T}+R_{k}^{(i)}+\bar{H}_{k}^{(i)}\left(A_{k}, C_{k}\right) \mathcal{U}_{k}^{(i)}, \quad k \geq 1 .
\end{gathered}
$$

Proof. Note that the local filtering algorithm has the same structure as the centralized filtering algorithm in Theorem 3.1. Therefore, the proof of Theorem 4.1 is thoroughly analogous to that of Theorem 3.1 and, consequently, it is omitted.

Once the local filters have been obtained, the distributed fusion filter, $\widehat{x}_{k / k}^{(D)}$, will be calculated as a matrix-weighted linear combination of the local filters, $\widehat{x}_{k / k}^{(i)}, i=$ $1, \ldots, m$, in which the weight matrices are computed by minimizing the mean squared estimation error. The distributed filters, $\widehat{x}_{k / k}^{(D)}$, and a formula for the error covariance matrices, $\Sigma_{k / k}^{(D)} \equiv E\left[\left(x_{k}-\widehat{x}_{k / k}^{(D)}\right)\left(x_{k}-\widehat{x}_{k / k}^{(D)}\right)^{T}\right]$, are presented in the following theorem.

Theorem 4.2. Let $\widehat{X}_{k / k}=\left(\widehat{x}_{k / k}^{(1) T}, \ldots, \widehat{x}_{k / k}^{(m) T}\right)^{T}$ be the vector constituted by the local filters calculated from the algorithm in Theorem 4.1; then, the distributed filtering 
estimators and their error covariance matrices are given by

$$
\begin{gathered}
\widehat{x}_{k / k}^{(D)}=\mathbf{\Upsilon}_{k / k} \boldsymbol{\Xi}_{k / k}^{-1} \widehat{X}_{k / k}, \quad k \geq 1 \\
\Sigma_{k / k}^{(D)}=A_{k} B_{k}^{T}-\mathbf{\Upsilon}_{k / k} \boldsymbol{\Xi}_{k / k}^{-1} \Upsilon_{k / k}^{T}, \quad k \geq 1 .
\end{gathered}
$$

with $\boldsymbol{\Xi}_{k / k} \equiv\left(K_{k / k}^{\widehat{x}^{(i j)}}\right)_{i, j=1, \ldots, m}$ and $\boldsymbol{\Upsilon}_{k / k} \equiv\left(K_{k / k}^{\widehat{x}^{(1)}}, \ldots, K_{k / k}^{\widehat{x}^{(m)}}\right)$, where the matrices $K_{k / k}^{\widehat{x}^{(i j)}} \equiv E\left[\widehat{x}_{k / k}^{(i)} \widehat{x}_{k / k}^{(j) T}\right], i, j=1, \ldots, m$, are obtained by

$$
K_{k / k}^{\widehat{x}^{(i j)}}=\left(A_{k}, C_{k}\right) K_{k}^{u^{(i j)}}\left(A_{k}, C_{k}\right)^{T}, \quad k \geq 1
$$

and the matrices $K_{k}^{u^{(i j)}} \equiv E\left[u_{k}^{(i)} u_{k}^{(j) T}\right]$ are given by

$$
K_{k}^{u^{(i j)}}=K_{k-1}^{u^{(i j)}}+\mathcal{U}_{k-1, k}^{(i j)} \Pi_{k}^{(j)-1} \mathcal{U}_{k}^{(j) T}+\mathcal{U}_{k}^{(i)} \Pi_{k}^{(i)-1} \mathcal{U}_{k}^{(j i) T}, \quad k \geq 1 ; \quad K_{0}^{u^{(i j)}}=0 .
$$

The matrices $\mathcal{U}_{h, k}^{(i j)} \equiv E\left[u_{h}^{(i)} \mu_{k}^{(j) T}\right], h=k-1, k$, are calculated by

$$
\begin{gathered}
\mathcal{U}_{k-1, k}^{(i j)}=\left(K_{k-1}^{u^{(i)}}-K_{k-1}^{u^{(i j)}}\right)\left(A_{k}, C_{k}\right)^{T} \bar{H}_{k}^{(j) T}, k \geq 1, \\
\mathcal{U}_{k}^{(i j)}=\mathcal{U}_{k-1, k}^{(i j)}+\mathcal{U}_{k}^{(i)} \Pi_{k}^{(i)-1} \Pi_{k}^{(i j)}, \quad k \geq 1 .
\end{gathered}
$$

Finally, the cross-covariance matrices, $\Pi_{k}^{(i j)} \equiv E\left[\mu_{k}^{(i)} \mu_{k}^{(j) T}\right]$, satisfy

$$
\begin{aligned}
\Pi_{k}^{(i j)}= & E\left[\widetilde{H}_{k}^{(i)} A_{k} B_{k}^{T} \widetilde{H}_{k}^{(j) T}\right]+D_{k}^{(i)} C_{k}^{T} \bar{H}_{k}^{(j) T}+R_{k}^{(i j)} \\
& +\bar{H}_{k}^{(i)}\left(A_{k}, C_{k}\right)\left(\mathcal{U}_{k}^{(j)}-\mathcal{U}_{k-1, k}^{(i j)}\right), \quad k \geq 1 .
\end{aligned}
$$

Proof. See Appendix B.

\section{Sequential fusion filtering estimators}

In the sequential fusion method, at each sampling time $k$, instead of processing the measurements of the different sensors as a vector, an algorithm is implemented to process sequentially these measurements. More precisely, the signal estimator based on the measurements of all the sensors up to time $k-1$ is sequentially updated using the successive measurements provided by every sensor at time $k$. Consider that the sensor measurements arrive at the processing center according to an arbitrary order and, without loss of generality, assume that this order is indicated by the sensor numbering; that is, for all $i=1, \ldots, m-1$, the observation $y_{k}^{(i)}$ from the $i$ th sensor is received before the observation $y_{k}^{(i+1)}$ from the $(i+1)$ th sensor. Hence, at each sampling time $k$, the update of the signal estimators is recursively carried out in real time according to the arriving order of measurements from the different sensors.

Our aim in this section is to use the sequential fusion method to obtain the LS linear filtering estimator, $\widehat{x}_{k / k}^{(S)}$, of the signal $x_{k}$ based on the observations made by all 
the sensors up to time $k$. The set of observations made by the first $i$ sensors at time $k$ will be denoted by $Y_{k}^{(i)}=\left\{y_{k}^{(1)}, \ldots, y_{k}^{(i)}\right\}$; hence, $Y_{k}^{(m)}$ is the set of observations made by all the sensors at time $k$, and $Y_{k}=\left\{Y_{1}^{(m)}, \ldots, Y_{k}^{(m)}\right\}$ are all the observations made by all the sensors up to time $k$. Also, for notational simplicity, we will write $Y_{k}^{(0)}$ to mean that no observations of any sensor are available at the instant $k$.

Clearly, $Y_{k}=\left\{y_{1}, \ldots, y_{k}\right\}$, with $y_{1}, \ldots, y_{k}$ the observations given in (4). Consequently, the sequential fusion filter is equal to the centralized one $\left(\widehat{x}_{k / k}^{(S)}=\widehat{x}_{k / k}^{(C)}\right)$ but, as it will be discussed in Remark 1, in comparison with the centralized fusion algorithm, the sequential fusion algorithm can significantly reduce the computational cost.

Let us denote $\widehat{x}_{k / k, i}$ the LS linear estimator of the signal $x_{k}$ based on the observations provided by all sensors up to time $k-1$ and the observations provided by the first $i$ sensors at time $k$; that is, the estimator of $x_{k}$ based on $\left\{Y_{k-1}, Y_{k}^{(i)}\right\}$. Since $Y_{k}=$ $\left\{Y_{k-1}, Y_{k}^{(m)}\right\}$, it is clear that the sequential fusion filter is given by $\widehat{x}_{k / k}^{(S)}=\widehat{x}_{k / k, m}$; so, for each $k \geq 1$, our aim is to derive a recursive algorithm to obtain sequentially the estimators $\widehat{x}_{k / k, 1}, \ldots, \widehat{x}_{k / k, m}$.

As in the previous centralized and local estimators, the derivation of the filtering estimators by the sequential fusion method will also be carried out using an innovation approach.

Innovation-based expression of the estimators. For any fixed $k$, let $\widehat{y}_{k / k, i-1}^{(i)}$ be the estimator of $y_{k}^{(i)}$ based on $\left\{Y_{k-1}, Y_{k}^{(i-1)}\right\}$. Note that this estimator is the part of the observation $y_{k}^{(i)}$ determined by the knowledge of the set $\left\{Y_{k-1}, Y_{k}^{(i-1)}\right\}$; then, the difference vector $\mu_{k, i} \equiv y_{k}^{(i)}-\widehat{y}_{k / k, i-1}^{(i)}$ is the new information or the innovation provided by $y_{k}^{(i)}$.

Using that the LS linear estimator based on the observations is equal to the one based on the innovations, we have that, for $i=0, \ldots, m$, the LS linear estimator, $\widehat{\xi}_{k / k, i}$, of a random vector $\xi_{k}$ based on the observations $\left\{Y_{k-1}, Y_{k}^{(i)}\right\}$, can be expressed as follows:

$$
\widehat{\xi}_{k / k, i}=\left(1-\delta_{k, 1}\right) \sum_{h=1}^{k-1} \sum_{j=1}^{m} E\left[\xi_{k} \mu_{h, j}^{T}\right] \Pi_{h, j}^{-1} \mu_{h, j}+\left(1-\delta_{i, 0}\right) \sum_{j=1}^{i} E\left[\xi_{k} \mu_{k, j}^{T}\right] \Pi_{k, j}^{-1} \mu_{k, j},
$$

where $\Pi_{h, j} \equiv E\left[\mu_{h, j} \mu_{h, j}^{T}\right], h \leq k$, denotes the covariance matrix of $\mu_{h, j}$.

Observation estimator $\widehat{y}_{k / k, i-1}^{(i)}$. To obtain an expression for $\widehat{y}_{k / k, i-1}^{(i)}$, we will start from (1) which, taking into account that $H_{k}^{(i)}$ is correlated with $H_{k}^{(j)}$ and, hence, correlated with the innovations $\mu_{k, j}$, for $j=1, \ldots, i-1$, is rewritten as

$$
y_{k}^{(i)}=\bar{H}_{k}^{(i)} x_{k}+\widetilde{H}_{k}^{(i)} x_{k}+v_{k}^{(i)}, \quad k \geq 1 .
$$

Clearly, the estimator of $\bar{H}_{k}^{(i)} x_{k}$ based on $\left\{Y_{k-1}, Y_{k}^{(i-1)}\right\}$ is $\bar{H}_{k}^{(i)} \widehat{x}_{k / k, i-1}$ and, to obtain the estimator of the vector $\widetilde{H}_{k}^{(i)} x_{k}+v_{k}^{(i)}$, we apply the general expression (18); so, 
taking into account that $E\left[\left(\widetilde{H}_{k}^{(i)} x_{k}+v_{k}^{(i)}\right) \mu_{h, j}^{T}\right]=0$, for $h \leq k-1$ and $j=1, \ldots, m$, and denoting $\mathcal{V}_{k, j}^{(i)} \equiv E\left[\left(\widetilde{H}_{k}^{(i)} x_{k}+v_{k}^{(i)}\right) \mu_{k, j}^{T}\right]$, we obtain

$$
\widehat{y}_{k / k, i-1}^{(i)}=\bar{H}_{k}^{(i)} \widehat{x}_{k / k, i-1}+\left(1-\delta_{i, 1}\right) \sum_{j=1}^{i-1} \mathcal{V}_{k, j}^{(i)} \Pi_{k, j}^{-1} \mu_{k, j}, \quad i=1, \ldots, m .
$$

Signal estimator $\widehat{x}_{k / k, i}$. From the general expression (18), to obtain the LS linear estimator $\widehat{x}_{k / k, i}$ it is necessary to calculate the coefficients

$$
\mathcal{X}_{k, h, j} \equiv E\left[x_{k} \mu_{h, j}^{T}\right]=E\left[x_{k} y_{h}^{(j) T}\right]-E\left[x_{k} \widehat{y}_{h / h, j-1}^{(j) T}\right], \quad h \leq k, \quad j=1, \ldots, m .
$$

Using (2) for $E\left[x_{k} y_{h}^{(j) T}\right]$ and expression (19) for $\widehat{y}_{h / h, j-1}^{(j)}$ together with (18) for $\widehat{x}_{h / h, j-1}$, we obtain that these coefficients are expressed as

$$
\mathcal{X}_{k, h, j}=\left(A_{k}, C_{k}\right) \mathcal{U}_{h, j}, \quad h \leq k, \quad j=1, \ldots, m,
$$

where

$$
\begin{aligned}
\mathcal{U}_{h, j}= & \left(\bar{H}_{h}^{(j)} B_{h}, D_{h}^{(j)}\right)^{T}-\left(1-\delta_{h, 1}\right) \sum_{h=1}^{h-1} \sum_{j^{\prime}=1}^{m} \mathcal{U}_{h^{\prime}, j^{\prime}} \Pi_{h^{\prime}, j^{\prime}}^{-1} \mathcal{U}_{h^{\prime}, j^{\prime}}^{T}\left(A_{h}, C_{h}\right)^{T} \bar{H}_{h}^{(j) T} \\
& -\left(1-\delta_{j, 1}\right) \sum_{j^{\prime}=1}^{j-1} \mathcal{U}_{h, j^{\prime}} \Pi_{h, j^{\prime}}^{-1}\left(\bar{H}_{h}^{(j)}\left(A_{h}, C_{h}\right) \mathcal{U}_{h, j^{\prime}}+\mathcal{V}_{h, j^{\prime}}^{(j)}\right)^{T}, \quad h \geq 1, \quad j=1, \ldots, m .
\end{aligned}
$$

Then, by defining the vectors

$$
\begin{gathered}
u_{k, i} \equiv \sum_{j=1}^{i} \mathcal{U}_{k, j} \Pi_{k, j}^{-1} \mu_{k, j}, \quad i=1, \ldots, m, \quad u_{k, 0} \equiv 0, \quad k \geq 1 \\
\mathbf{U}_{k} \equiv \sum_{h=1}^{k} u_{h, m}, \quad k \geq 1 ; \quad \mathbf{U}_{0} \equiv 0
\end{gathered}
$$

and applying the expressions (18), (20) and (21), it is easy to see that the estimator is given by $\widehat{x}_{k / k, i}=\left(A_{k}, C_{k}\right)\left(\mathbf{U}_{k-1}+u_{k, i}\right)$.

Taking into account the above results, the recursive algorithm for the LS linear filter, under the sequential fusion method, is derived in the following theorem.

Theorem 5.1. Under assumptions (A1)-(A5), the sequential fusion filter $\widehat{x}_{k / k}^{(S)}$ and the filtering error covariance matrix, $\Sigma_{k / k}^{(S)} \equiv E\left[\left(x_{k}-\widehat{x}_{k / k}^{(S)}\right)\left(x_{k}-\widehat{x}_{k / k}^{(S)}\right)^{T}\right]$ are given by

$$
\widehat{x}_{k / k}^{(S)}=\widehat{x}_{k / k, m}, \quad \Sigma_{k / k}^{(S)}=\Sigma_{k / k, m}, \quad k \geq 1,
$$

where, for each $k \geq 1$, the estimator $\widehat{x}_{k / k, m}$ and its error covariance matrix, $\Sigma_{k / k, m} \equiv$ $E\left[\left(x_{k}-\widehat{x}_{k / k, m}\right)\left(x_{k}-\widehat{x}_{k / k, m}\right)^{T}\right]$, are sequentially obtained by the following recursive 
algorithm:

$$
\begin{gathered}
\widehat{x}_{k / k, i}=\left(A_{k}, C_{k}\right)\left(\mathbf{U}_{k-1}+u_{k, i}\right), \quad i=1, \ldots, m \\
\Sigma_{k / k, i}=A_{k} B_{k}^{T}-\left(A_{k}, C_{k}\right)\left(\mathbf{K}_{k-1}^{\mathbf{U}}+K_{k, i}^{u}\right)\left(A_{k}, C_{k}\right)^{T}, \quad i=1, \ldots, m .
\end{gathered}
$$

The vectors $u_{k, i}$ and the matrices $K_{k, i}^{u} \equiv E\left[u_{k, i} u_{k, i}^{T}\right]$ are obtained from

$$
\begin{gathered}
u_{k, i}=u_{k, i-1}+\mathcal{U}_{k, i} \Pi_{k, i}^{-1} \mu_{k, i}, \quad i=1, \ldots, m ; \quad u_{k, 0}=0 \\
K_{k, i}^{u}=K_{k, i-1}^{u}+\mathcal{U}_{k, i} \Pi_{k, i}^{-1} \mathcal{U}_{k, i}^{T}, \quad i=1, \ldots, m ; \quad K_{k, 0}^{u}=0
\end{gathered}
$$

and the vectors $\mathbf{U}_{k}$ and the matrices $\mathbf{K}_{k}^{\mathbf{U}} \equiv E\left[\mathbf{U}_{k} \mathbf{U}_{k}^{T}\right]$ satisfy

$$
\begin{array}{cl}
\mathbf{U}_{k}=\mathbf{U}_{k-1}+u_{k, m}, \quad k \geq 1 ; & \mathbf{U}_{0}=0 \\
\mathbf{K}_{k}^{\mathbf{U}}=\mathbf{K}_{k-1}^{\mathbf{U}}+K_{k, m}^{u}, \quad k \geq 1 ; & \mathbf{K}_{0}^{\mathbf{U}}=0 .
\end{array}
$$

The matrices $\mathcal{U}_{k, i} \equiv E\left[u_{k, i} \mu_{k, i}^{T}\right]$ are given by

$$
\begin{aligned}
\mathcal{U}_{k, i}= & \left(\bar{H}_{k}^{(i)} B_{k}, D_{k}^{(i)}\right)^{T}-\left(\mathbf{K}_{k-1}^{\mathbf{U}}+K_{k, i-1}^{u}\right)\left(A_{k}, C_{k}\right)^{T} \bar{H}_{k}^{(i) T} \\
& -\left(1-\delta_{i, 1}\right) \sum_{j=1}^{i-1} \mathcal{U}_{k, j} \Pi_{k, j}^{-1} \mathcal{V}_{k, j}^{(i) T}, \quad i=1, \ldots, m .
\end{aligned}
$$

The innovation, $\mu_{k, i}=y_{k}^{(i)}-\widehat{y}_{k / k, i-1}^{(i)}$, and its covariance matrix, $\Pi_{k, i}=E\left[\mu_{k, i} \mu_{k, i}^{T}\right]$, are obtained by

$$
\begin{gathered}
\mu_{k, i}=y_{k}^{(i)}-\bar{H}_{k}^{(i)}\left(A_{k}, C_{k}\right)\left(\mathbf{U}_{k-1}+u_{k, i-1}\right)-\left(1-\delta_{i, 1}\right) \sum_{j=1}^{i-1} \mathcal{V}_{k, j}^{(i)} \Pi_{k, j}^{-1} \mu_{k, j}, \quad i=1, \ldots, m, \\
\Pi_{k, i}=\bar{H}_{k}^{(i)}\left(A_{k}, C_{k}\right) \mathcal{U}_{k, i}+\mathcal{V}_{k, i}^{(i)}, \quad i=1, \ldots, m .
\end{gathered}
$$

The matrices $\mathcal{V}_{k, j}^{(i)}=E\left[\left(\widetilde{H}_{k}^{(i)} x_{k}+v_{k}^{(i)}\right) \mu_{k, j}^{T}\right]$ satisfy

$$
\begin{aligned}
\mathcal{V}_{k, j}^{(i)}= & E\left[\widetilde{H}_{k}^{(i)} A_{k} B_{k}^{T} \widetilde{H}_{k}^{(j) T}\right]-\left(1-\delta_{j, 1}\right) \sum_{j^{\prime}=1}^{j-1} \mathcal{V}_{k, j^{\prime}}^{(i)} \Pi_{k, j^{\prime}}^{-1}\left(\bar{H}_{k}^{(j)}\left(A_{k}, C_{k}\right) \mathcal{U}_{k, j^{\prime}}+\mathcal{V}_{k, j^{\prime}}^{(j)}\right)^{T} \\
& +D_{k}^{(i)} C_{k}^{T} \bar{H}_{k}^{(j) T}+R_{k}^{(i, j)}, \quad i=1, \ldots, m, \quad j=1, \ldots, i
\end{aligned}
$$

Proof. See Appendix C.

As it has been previously indicated, since $Y_{k}=\left\{y_{1}, \ldots, y_{k}\right\}$ with $y_{1}, \ldots, y_{k}$ the observations given in (4), the sequential fusion filter is equal to the centralized one. Actually, the following Theorem provides the proof of this equivalence between the least-squares centralized fusion and least-squares sequential fusion algorithms proposed in this paper. 
Theorem 5.2. Under assumptions (A1)-(A5), the estimation accuracy of the centralized fusion filter given in Theorem 3.1 and that of the sequential fusion filter given in Theorem 5.1 are equivalent under the same initial values; in fact, $\widehat{x}_{k / k}^{(C)}=\widehat{x}_{k / k}^{(S)}$ and $\Sigma_{k / k}^{(C)}=\Sigma_{k / k}^{(S)}$, for all $k \geq 1$.

Proof. See Appendix D.

Remark 1. Computational complexity. The implementation efficiency of an algorithm is expressed by its computational complexity, measured by the amount of work (number of operations) performed at each iteration. Such complexity is usually expressed using the $\mathcal{O}(\cdot)$ notation which, omitting multiplicative constants and lower order terms, represents the order of magnitude as a function of the size of the input data. In our study, we have considered a signal of dimension $n_{x}$ and observations coming from $m$ sensors, all of dimension $n_{y}$.

- Centralized fusion filtering algorithm. The dimension of the stacked observations (4), containing the measurements of all the sensors, is $n_{y} m$; then, at each iteration, the centralized fusion algorithm, given in Theorem 3.1, requires the computation of the inverse of the innovation covariance matrix (of dimension $n_{y} m \times n_{y} m$ ) and, hence, the computational complexity of this algorithm has the order of magnitude $\mathcal{O}\left(\left(n_{y} m\right)^{3}\right)$

- Sequential fusion filtering algorithm. In the sequential fusion algorithm given in Theorem 5.1, at each iteration $k$, the innovations $\mu_{k, i}, i=2, \ldots, m$, given by (26), require the computation of $i-1$ products of $n_{y} \times n_{y}$ matrices; namely, $\mathcal{V}_{k, j}^{(i)} \Pi_{k, j}^{-1}$, for $j=1, \ldots, i-1$. Since each of these products has the order of magnitude $\mathcal{O}\left(n_{y}^{3}\right)$ and we have $1+2+\cdots+m-1=\frac{m(m-1)}{2}$ products, the computational complexity of the proposed sequential fusion algorithm has the order of magnitude $\mathcal{O}\left(\frac{m(m-1)}{2} n_{y}^{3}\right)$.

So, compared with the centralized fusion algorithm, the sequential fusion one can provide a significant reduction of the computational cost even for a small number of sensors; clearly, the reduction is greater when the number of sensors, $m$, increases.

Remark 2. Centralized, distributed and sequential fusion filtering algorithms have been obtained when the sensor measurement model includes random parameter matrices and additive noises that are cross-correlated among the different sensors and correlated with the signal at the same and future time steps. In contrast to the methodology based on the state-space model used in Lin and Sun (2018) and Lin and Sun (2019), a covariance-based approach is used here to derive the algorithms. In similarity with these papers, the projection theory is used in the algorithm design and the computational cost of the proposed algorithms is the same as that of the algorithms in Lin and Sun (2018) and Lin and Sun (2019).

\section{Numerical simulation example}

In this section, the application of the centralized, distributed and sequential filtering algorithms proposed in the current paper is illustrated by a simulation example. 
Consider a two-dimensional signal whose first and second component represent the position and velocity of a target, respectively. As in Lin and Sun (2018), assume that the signal evolution equation is given by:

$$
x_{k+1}=\left(F+\varepsilon_{k}^{(1)} F_{1}+\varepsilon_{k}^{(2)} F_{2}\right) x_{k}+\varrho w_{k}, \quad k \geq 1,
$$

where

$$
F=\left(\begin{array}{cc}
0.95 & 0.1 \\
0 & 0.95
\end{array}\right), F_{1}=\left(\begin{array}{cc}
0.1 & 0 \\
0 & 0.01
\end{array}\right), F_{2}=\left(\begin{array}{cc}
0.2 & 0 \\
0 & 0.02
\end{array}\right), \varrho=\left(\begin{array}{l}
0.8 \\
0.6
\end{array}\right)
$$

The sequences $\left\{\varepsilon_{k}^{(1)}\right\}_{k \geq 1},\left\{\varepsilon_{k}^{(2)}\right\}_{k \geq 1}$ and $\left\{w_{k}\right\}_{k \geq 1}$ are standard white gaussian scalar noises. The initial signal $x_{0}$ is a two-dimensional zero-mean gaussian random vector with covariance matrix $E\left[x_{0} x_{0}^{T}\right]=0.1 I$.

Assuming that the initial signal, $x_{0}$, and the sequences $\left\{\varepsilon_{k}^{(1)}\right\}_{k>1},\left\{\varepsilon_{k}^{(2)}\right\}_{k>1}$ and $\left\{w_{k}\right\}_{k \geq 1}$ are mutually independent, the signal covariance function is given by $E\left[x_{k} x_{h}^{T}\right]=F^{k-h} E\left[x_{h} x_{h}^{T}\right], h \leq k$, where $E\left[x_{h} x_{h}^{T}\right], h \geq 1$, is recursively obtained by:

$$
E\left[x_{h} x_{h}^{T}\right]=F E\left[x_{h-1} x_{h-1}^{T}\right] F^{T}+F_{1} E\left[x_{h-1} x_{h-1}^{T}\right] F_{1}^{T}+F_{2} E\left[x_{h-1} x_{h-1}^{T}\right] F_{2}^{T}+\varrho \varrho^{T} .
$$

Hence, assumption (A1) is satisfied just taking, for example, $A_{k}=F^{k}$ and $B_{h}^{T}=$ $F^{-h} E\left[x_{h} x_{h}^{T}\right]$.

The measurements of the target are provided by four sensors, which are affected by different uncertainties: continuous gain degradation with correlation at the same time instant is considered in sensors 1 and 4, discrete gain degradation in sensor 2 and missing measurements in sensor 3. Specifically, we consider the model (1) for the measurements $\left\{y_{k}^{(i)}\right\}_{k>1}, i=1,2,3,4$, with $H_{k}^{(1)}=\theta_{k}^{(1)}(0.75,1), H_{k}^{(2)}=\theta_{k}^{(2)}(1,0.75)$, $H_{k}^{(3)}=\theta_{k}^{(3)}(1,1)$ and $H_{k}^{(4)}=\theta_{k}^{(1)}(1,1.25)$, where $\left\{\theta_{k}^{(i)}\right\}_{k>1}, i=1,2,3$, are mutually independent sequences of independent random variables with the following timeinvariant probability distributions:

- $\theta_{k}^{(1)}$ is uniformly distributed over $[0.2,0.8]$.

- $P\left[\theta_{k}^{(2)}=0\right]=0.3, \quad P\left[\theta_{k}^{(2)}=0.5\right]=0.3, \quad P\left[\theta_{k}^{(2)}=1\right]=0.4$.

- $\theta_{k}^{(3)}$ is a Bernoulli random variable with $P\left[\theta_{k}^{(3)}=1\right]=\bar{\theta}$.

The additive noise processes $\left\{v_{k}^{(i)}\right\}_{k \geq 1}, i=1,2,3,4$, are defined by $v_{k}^{(i)}=c_{i} w_{k-1}$, with $c_{i}=10 i$ for $i=1,2,3,4$. Clearly, these noises are correlated, with $E\left[v_{k}^{(i)} v_{h}^{(j)}\right]=$ $c_{i} c_{j} \delta_{k, h}, \quad i, j=1,2,3,4$; hence, assumption (A2) holds with $R_{k}^{(i j)}=c_{i} c_{j}, k \geq 1$. Also, it is clear that $E\left[w_{k-1} v_{h}^{(i)}\right]=c_{i} \delta_{k, h}$ and, as a result, the signal process and the observation noises are correlated with cross-covariance functions given by $E\left[x_{k} v_{h}^{(i)}\right]=$ $F^{k-h} E\left[x_{h} v_{h}^{(i)}\right]=F^{k-h} E\left[\varrho w_{h-1} v_{h}^{(i)}\right]=c_{i} F^{k-h} \varrho$, for $h \leq k$, and $E\left[x_{k} v_{h}^{(i)}\right]=0$, for $h>k$; hence, assumption (A3) is satisfied just taking $C_{k}=F^{k}$ and $D_{h}^{(i) T}=c_{i} F^{-h} \varrho$.

To illustrate and compare the effectiveness of the local and fusion estimators, the proposed algorithms were implemented using MATLAB and two hundred iterations were performed for the centralized, distributed and sequential fusion filtering algo- 


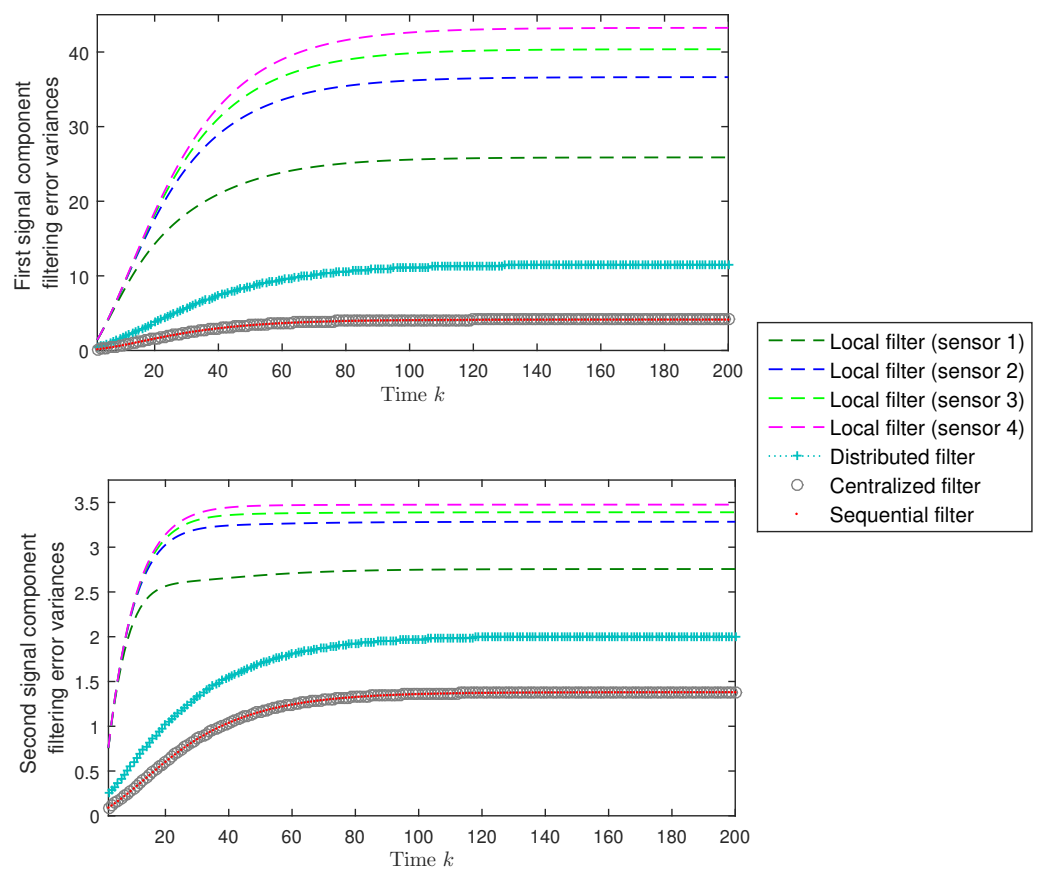

Figure 1. Error variance comparison of the local, distributed, centralized and sequential filtering estimators when $\bar{\theta}=0.5$.

rithms, besides the local filtering algorithms.

(1) Estimation accuracy. In order to compare the accuracy of the proposed fusion estimators, the error variances of the local filters and the different fusion filters were calculated. In Figure 1, considering $\bar{\theta}=0.5$, the error variances of these filtering estimators are displayed; this figure shows, on the one hand, that, as expected, the distributed fusion filtering estimators outperform all the local ones (that is, the error variances of the distributed fusion estimators are smaller than those of every local filter) and, on the other hand, that the error variances corresponding to the distributed fusion filters are greater than those of the centralized and sequential filters. In Figure 1 it is also observed that, as indicated in Section 5, the accuracy of the sequential filter is equal to that of the centralized filter.

(2) Tracking performance of the centralized and sequential fusion estimates. Using simulated values of the signal and the observations in the four sensors, both centralized and sequential fusion filtering estimates were calculated when $\bar{\theta}=0.5$. For both signal components (position and velocity), Figure 2 shows a satisfactory and efficient tracking performance of these fusion filtering estimates which, corroborating what we indicated in Section 5, are exactly the same.

(3) Mean-squared error comparison. The distributed, centralized and sequential fusion estimators have also been compared, when $\bar{\theta}=0.5$, using the filtering mean-squared error at each time instant $k, \mathrm{MSE}_{k}=\frac{1}{1000} \sum_{r=1}^{1000}\left(x_{k}^{(r)}-\widehat{x}_{k / k}^{(r)}\right)^{2}$, where $\left\{x_{k}^{(r)}\right\}_{1 \leq k \leq 200}$ is the $r$-th set of simulated signal data and $\widehat{x}_{k / k}^{(r)}$ is the centralized/sequential filter 

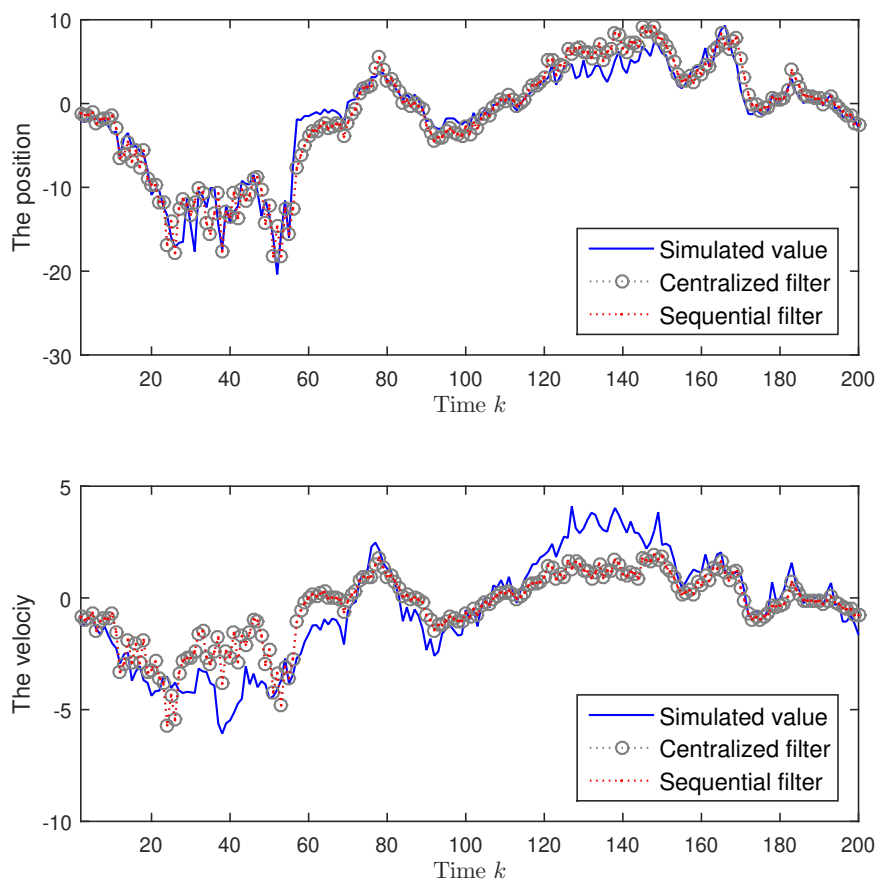

Figure 2. Simulated values together with centralized and sequential fusion filtering estimates when $\bar{\theta}=0.5$.

at the sampling time $k$ in the $r$-th simulation run. The results are displayed in Figure 3, from which analogous conclusions to those in the above figures can be drawn.

(4) Influence of the missing probabilities. As it has been previously indicated, randomly missing measurements are assumed to occur in sensor 3 with probability $1-\bar{\theta}$. In order to show the effect of this missing measurement phenomenon, the centralized/sequential filtering error variances are plotted in Figure 4 for different values of the probability $\bar{\theta}$; namely, we assumed that $\bar{\theta}$ varies from 0.1 to 0.9 . From this figure, we observe that, as expected, the centralized/sequential error variances become smaller as $\bar{\theta}$ increases. Consequently, the performance of these filters is indeed influenced by this probability, improving when the probability of missing measurements, $1-\bar{\theta}$, decreases. Finally, it is also inferred that this improvement is more appreciable when $\bar{\theta} \geq 0.5$.

\section{Conclusion}

Three different approaches (centralized, distributed and sequential) to the LS fusion filtering problem have been investigated in this paper for discrete-time stochastic signals, which are observed by a multisensor network system whose measurements are impaired by random uncertainties modelled by random parameter matrices. As it is known, the centralized fusion estimation, despite theoretically providing optimal estimators, suffers from high computational complexity. This and other disadvantages can be overcome by using the distributed fusion estimation method which, on the other 

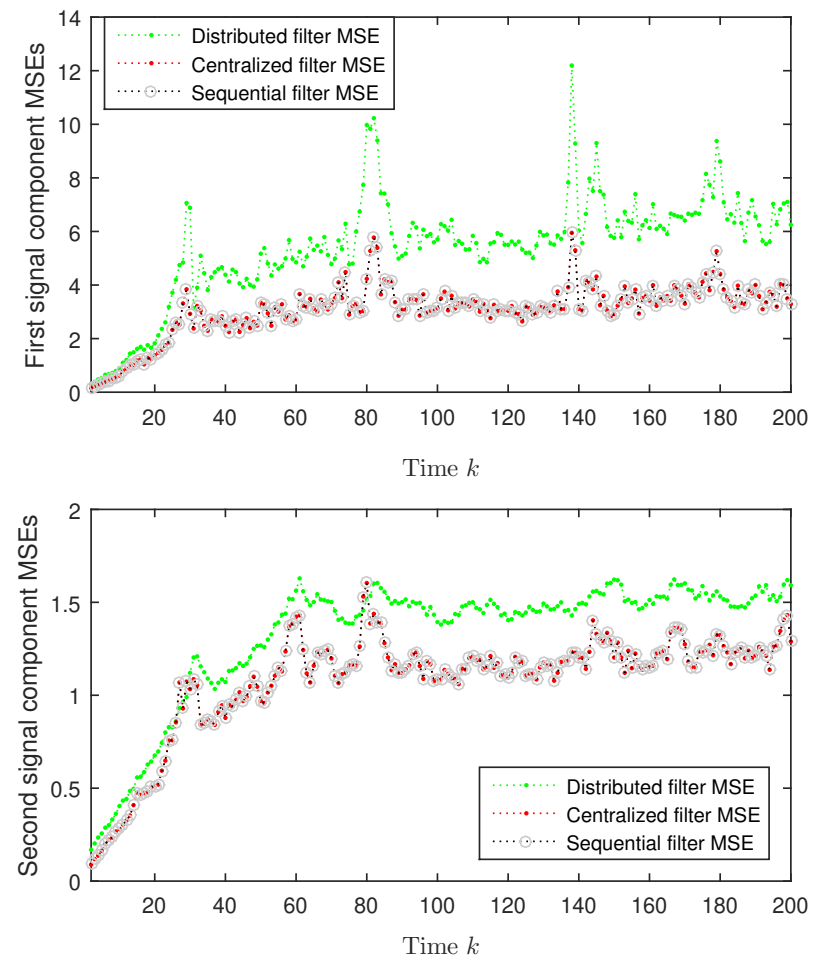

Figure 3. Distributed, centralized and sequential filtering mean-squared errors (MSE) when $\bar{\theta}=0.5$.

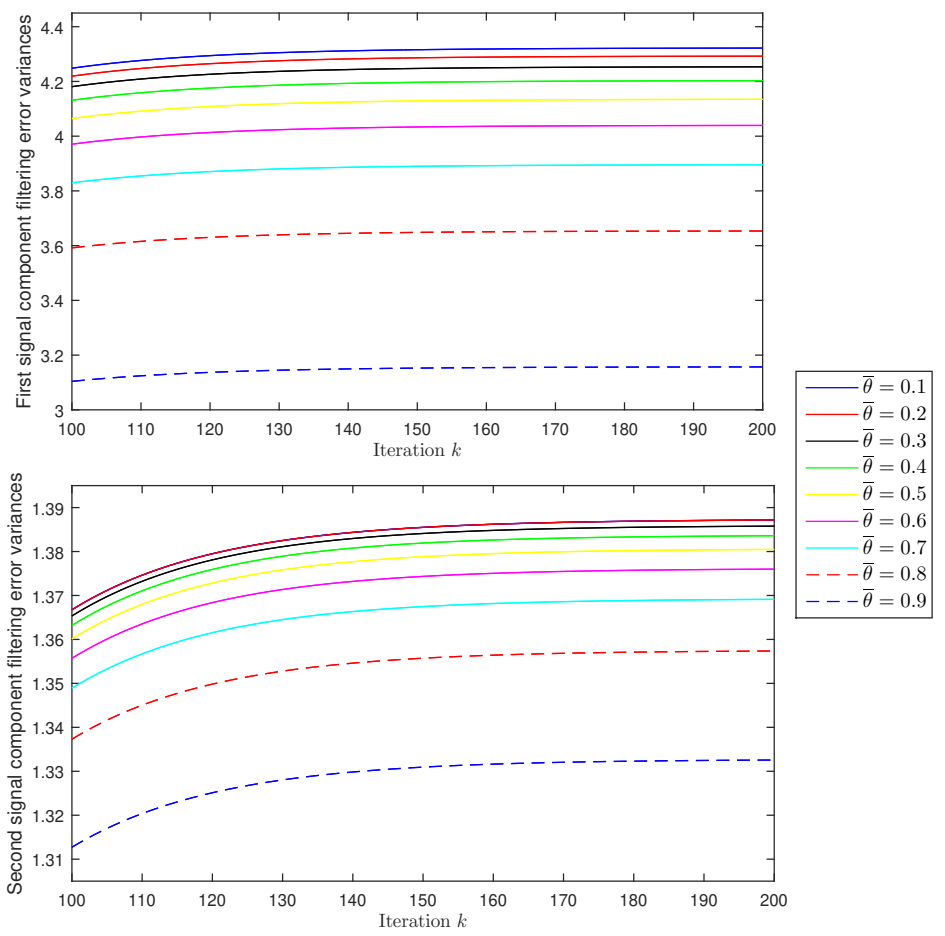

Figure 4. Centralized/Sequential filtering error variances for $\bar{\theta}$ from 0.1 to 0.9 . 
side, may provide suboptimal estimators. The sequential fusion method has the same estimation accuracy as the centralized one, thus providing optimal estimators as well, but the sensor data are processed in a sequential way, which yields a considerable reduction of the computational cost. Most sequential fusion estimation algorithms in the literature require the knowledge of the signal evolution model. However, in many practical applications, such information may not be available and only the first and second-order moments of the signal and the processes involved in the sensor measurement equations can be obtained.

This paper has addressed the centralized, distributed and sequential fusion estimation problems using covariance information when the sensor measurement model includes random parameter matrices and additive noises that are cross-correlated across sensors and correlated with the signal at the same and future time steps. A simulation example has shown how state-space models with additive noises correlated with the system noise at the previous time step fit the proposed covariance-based approach and how multiple uncertainties in the measurement equations (namely, discrete or continuous gain degradation and missing measurements) are covered by the current measurement model with random parameter matrices. The simulation results have illustrated how the proposed fusion filters perform and how the missing measurement phenomenon influences the estimation accuracy.

\section{Disclosure statement}

No potential conflict of interest was reported by the authors.

\section{Funding}

This research is supported by Ministerio de Economía, Industria y Competitividad, Agencia Estatal de Investigación and Fondo Europeo de Desarrollo Regional FEDER (grant no. MTM2017-84199-P).

\section{Notes on contributors}

R. Caballero-Águila received her MSc degree in Mathematics (Statistics) from the University of Granada (Spain) in 1997 and her PhD in Polynomial Filtering in Systems with Uncertain Observations in 1999. In 1997, she joined the University of Jaén (Spain), where she is now associate professor at the Department of Statistics and Operations Research. Her research interest is mainly focused on stochastic systems, filtering, prediction and smoothing.

A. Hermoso-Carazo received her MSc degree in Mathematics (Statistics) and her PhD in Likelihood Test in Log Normal Processes, both from the University of Granada (Spain) in 1979 and 1984, respectively. She is currently a professor at the Department of Statistics and Operations Research, University of Granada (Spain). Her current research interests include stochastic systems, filtering and prediction.

J. Linares-Pérez received her MSc degree in Mathematics (Statistics) and her PhD in Stochastic Differential Equations, both from the University of Granada (Spain) in 1980 and 1982, respectively. She is currently a professor at the Department of Statistics and 
Operations Research, University of Granada (Spain). Her research interest involves the areas of stochastic calculus and estimation in stochastic systems.

\section{References}

Caballero-Águila, R., Hermoso-Carazo, A., and Linares-Pérez, J. (2015). Optimal state estimation for networked systems with random parameter matrices, correlated noises and delayed measurements. International Journal of General Systems, 44(2):142-154.

Caballero-Águila, R., Hermoso-Carazo, A., and Linares-Pérez, J. (2016). Fusion estimation using measured outputs with random parameter matrices subject to random delays and packet dropouts. Signal Processing, 127:12-23.

Caballero-Águila, R., Hermoso-Carazo, A., and Linares-Pérez, J. (2017). Covariance-based fusion filtering for networked systems with random transmission delays and non-consecutive losses. International Journal of General Systems, 46(7):752-771.

Caballero-Águila, R., Hermoso-Carazo, A., and Linares-Pérez, J. (2018). Centralized fusion approach to the estimation problem with multi-packet processing under uncertainty in outputs and transmissions. Sensors, 18(8):2697.

Caballero-Águila, R., Hermoso-Carazo, A., and Linares-Pérez, J. (2019a). Networked distributed fusion estimation under uncertain outputs with random transmission delays, packet losses and multi-packet processing. Signal Processing, 156:71-83.

Caballero-Águila, R., Hermoso-Carazo, A., Linares-Pérez, J., and Wang, Z. (2019b). A new approach to distributed fusion filtering for networked systems with random parameter matrices and correlated noises. Information Fusion, 45:324-332.

Ding, D., Wang, Z., and Shen, B. (2014). Recent advances on distributed filtering for stochastic systems over sensor networks. International Journal of General Systems, 43(3-4):372-386.

Dong, H., Wang, Z., Alsaadi, F. E., and Ahmad, B. (2015). Event-triggered robust distributed state estimation for sensor networks with state-dependent noises. International Journal of General Systems, 44(2):254-266.

Feng, X., Wu, L., and Wen, C. (2018). Sequential fault-tolerant fusion estimation for multisensor time-varying systems. Discrete Dynamics in Nature and Society, ID 4504206:9 pages.

Hu, J., Wang, Z., Alsaadi, F. E., and Hayat, T. (2017). Event-based filtering for time-varying nonlinear systems subject to multiple missing measurements with uncertain missing probabilities. Information Fusion, 38:74-83.

Kailath, T., Sayed, A. H., and Hassibi, B. (2000). Linear estimation. Prentice Hall, Upper Saddle River, New Jersey.

Kettner, A. M. and Paolone, M. (2017). Sequential discrete kalman filter for real-time state estimation in power distribution systems: Theory and implementation. IEEE Transactions on Instrumentation and Measurement, 66(9):2358-2370.

Li, W., Jia, Y., and Du, J. (2017). Distributed filtering for discrete-time linear systems with fading measurements and time-correlated noise. Digital Signal Processing, 60:211-219.

Li, X. (2003). Optimal linear estimation fusion, part vii: Dynamic systems. In Proceedings of the 6th International Conference of Information Fusion. IEEE.

Lin, H. and Sun, S. (2018). Optimal sequential fusion estimation with stochastic parameter perturbations, fading measurements, and correlated noises. IEEE Transactions on Signal Processing, 66(13):3571-3583.

Lin, H. and Sun, S. (2019). Globally optimal sequential and distributed fusion state estimation for multi-sensor systems with cross-correlated noises. Automatica, 101:128-137.

Liu, D., Liu, Y., and Alsaadi, F. E. (2016a). A new framework for output feedback controller design for a class of discrete-time stochastic nonlinear system with quantization and missing measurement. International Journal of General Systems, 45(5):517-531.

Liu, Y., Wang, Z., He, X., and Zhou, D. H. (2016b). Minimum-variance recursive filtering 
over sensor networks with stochastic sensor gain degradation: Algorithms and performance analysis. IEEE Transactions on Control of Network Systems, 3(3):265-274.

Ma, J. and Sun, S. (2013). Centralized fusion estimators for multisensor systems with random sensor delays, multiple packet dropouts and uncertain observations. IEEE Sensors Journal, 13:1228-1235.

Sun, S., Lin, H., Ma, J., and Li, X. (2017a). Multi-sensor distributed fusion estimation with applications in networked systems: A review paper. Information Fusion, 38:122-134.

Sun, S., Tian, T., and Lin, H. (2017b). State estimators for systems with random parameter matrices, stochastic nonlinearities, fading measurements and correlated noises. Information Sciences, 397-398:118-136.

Wang, W. and Zhou, J. (2017). Optimal linear filtering design for discrete-time systems with cross-correlated stochastic parameter matrices and noises. IET Control Theory and Applications, 11(18):3353-3362.

Wang, X. and Sun, S. (2017). Optimal recursive estimation for networked descriptor systems with packet dropouts, multiplicative noises and correlated noises. Aerospace Science and Technology, 63:41-53.

Wen, C., Cai, Y., Wen, C., and Xu, X. (2013). Optimal sequential Kalman filtering with cross-correlated measurement noises. Aerospace Science and Technology, 26:153-159.

Yan, L., Li, X. R., Xia, Y., and Fu, M. (2013). Optimal sequential and distributed fusion for state estimation in cross-correlated noise. Automatica, 49:3607-3612.

Yang, Y., Liang, Y., Pan, Q., Qin, Y., and Yang, F. (2016). Distributed fusion estimation with square-root array implementation for markovian jump linear systems with random parameter matrices and cross-correlated noises. Information Sciences, 370-3715:446-462.

Zhang, H., Hu, J., Zou, L., Yu, X., and Wu, Z. (2018). Event-based state estimation for timevarying stochastic coupling networks with missing measurements under uncertain occurrence probabilities. International Journal of General Systems, 47(5):506-521.

Zhou, F., Wu, L., and Feng, X. (2016). Sequential fusion for asynchronous multi-sensor fading measurements. International Journal of Control and Automation, 9(4):197-208.

Zhu, C., Xia, Y., Xie, L., and Yan, L. (2013). Optimal linear estimation for systems with transmission delays and packet dropouts. IET Signal Processing, 7(9):814-823.

\section{Appendix A. Proof of Theorem 3.1}

Let start by expressing the LS linear estimator of $x_{k}$ based on the observations $y_{h}, h \leq$ $L$, as a linear combination of the innovations $\mu_{h}, h \leq L$; namely:

$$
\widehat{x}_{k / L}^{(C)}=\sum_{h=1}^{L} E\left[x_{k} \mu_{h}^{T}\right] \Pi_{h}^{-1} \mu_{h} .
$$

Hence, to obtain the LS linear filter $\widehat{x}_{k / k}^{(C)}$, it is necessary to calculate the coefficients $\mathcal{X}_{k, h} \equiv E\left[x_{k} \mu_{h}^{T}\right]=E\left[x_{k} y_{h}^{T}\right]-E\left[x_{k} \widehat{y}_{h / h-1}^{(C) T}\right]$, for $h \leq k$.

Taking orthogonal projections, the centralized one-stage observation predictor $\widehat{y}_{h / h-1}^{(C)}$ is expressed as $\widehat{y}_{h / h-1}^{(C)}=\bar{H}_{h} \widehat{x}_{h / h-1}^{(C)}$. Then, using (5) for $E\left[x_{k} y_{h}^{T}\right]$ and (29) for $\widehat{x}_{h / h-1}^{(C)}$, we obtain that

$$
\mathcal{X}_{k, h}=\left(A_{k}, C_{k}\right) \mathcal{U}_{h}, \quad h \leq k,
$$


where $\mathcal{U}_{h}$ is a function satisfying

$$
\mathcal{U}_{h}=\left(\bar{H}_{h} B_{h}, D_{h}\right)^{T}-\left(1-\delta_{h, 1}\right) \sum_{j=1}^{h-1} \mathcal{U}_{j} \Pi_{j}^{-1} \mathcal{U}_{j}^{T}\left(A_{h}, C_{h}\right)^{T} \bar{H}_{h}^{T}, \quad h \geq 1
$$

Then, by defining the vectors $u_{k} \equiv \sum_{h=1}^{k} \mathcal{U}_{h} \Pi_{h}^{-1} \mu_{h}, k \geq 1$, and the matrices $K_{k}^{u} \equiv$ $E\left[u_{k} u_{k}^{T}\right]=\sum_{h=1}^{k} \mathcal{U}_{h} \Pi_{h}^{-1} \mathcal{U}_{h}^{T}, k \geq 1$, expressions (7)-(10) are easily derived.

To obtain expression (11) for $\Pi_{k}=E\left[\mu_{k} \mu_{k}^{T}\right]$, we use that $\Pi_{k}=E\left[y_{k} \mu_{k}^{T}\right]$ and, rewriting (4) as $y_{k}=\bar{H}_{k} x_{k}+\widetilde{H}_{k} x_{k}+v_{k}$, we have $E\left[y_{k} \mu_{k}^{T}\right]=\bar{H}_{k} E\left[x_{k} \mu_{k}^{T}\right]+E\left[\left(\widetilde{H}_{k} x_{k}+\right.\right.$ $\left.\left.v_{k}\right) \mu_{k}^{T}\right]$. Now, taking into account that the first expectation is $E\left[x_{k} \mu_{k}^{T}\right]=\mathcal{X}_{k, k}=$ $\left(A_{k}, C_{k}\right) \mathcal{U}_{k}$, and using (6) for $E\left[\left(\widetilde{H}_{k} x_{k}+v_{k}\right) \mu_{k}^{T}\right]=E\left[\left(\widetilde{H}_{k} x_{k}+v_{k}\right) y_{k}^{T}\right]$, expression (11) for $\Pi_{k}$ is derived.

\section{Appendix B. Proof of Theorem 4.2}

Expression (12) for the distributed filtering estimators and their error covariance matrices can be easily checked by applying the LS criterion (see e.g. Caballero-Águila et al. (2016)).

Expression (13) is straightforward just using that $\widehat{x}_{k / k}^{(i)}=\left(A_{k}, C_{k}\right) u_{k}^{(i)}$, and expressions (14) and (16) are obtained with no difficulty using the recursive formula of $u_{k}^{(i)}$ given in Theorem 4.1 .

Next, we derive (15) for $\mathcal{U}_{k-1, k}^{(i j)}=E\left[u_{k-1}^{(i)} y_{k}^{(j) T}\right]-E\left[u_{k-1}^{(i)} \widehat{y}_{k / k-1}^{(j) T}\right]$ as follows:

- $E\left[u_{k-1}^{(i)} y_{k}^{(j) T}\right]$ is obtained by using (1) for $y_{k}^{(j)}$ and taking into account that, by the projection theory, $E\left[u_{k-1}^{(i)} x_{k}^{T}\right]=E\left[u_{k-1}^{(i)} \widehat{x}_{k / k-1}^{(i) T}\right]$; so, since $\widehat{x}_{k / k-1}^{(i)}=\left(A_{k}, C_{k}\right) u_{k-1}^{(i)}$, we have $E\left[u_{k-1}^{(i)} y_{k}^{(j) T}\right]=K_{k-1}^{u^{(i)}}\left(A_{k}, C_{k}\right)^{T} \bar{H}_{k}^{(j) T}$.

- Using the expression of the observation predictor, $\widehat{y}_{k / k-1}^{(j)}=\bar{H}_{k}^{(j)}\left(A_{k}, C_{k}\right) u_{k-1}^{(j)}$, given in Theorem 4.1, we have that $E\left[u_{k-1}^{(i)} \widehat{y}_{k / k-1}^{(j) T}\right]=K_{k-1}^{u^{(i j)}}\left(A_{k}, C_{k}\right)^{T} \bar{H}_{k}^{(j) T}$.

From both expectations, expression (15) for $\mathcal{U}_{k-1, k}^{(i j)}$ is proven.

Finally, we derive expression (17) for $\Pi_{k}^{(i j)}=E\left[y_{k}^{(i)} \mu_{k}^{(j) T}\right]-E\left[\widehat{y}_{k / k-1}^{(i)} \mu_{k}^{(j) T}\right]$. On the one hand, by an analogous reasoning to that used for (11) but using now (3), we obtain

$$
E\left[y_{k}^{(i)} \mu_{k}^{(j) T}\right]=E\left[\widetilde{H}_{k}^{(i)} A_{k} B_{k}^{T} \widetilde{H}_{k}^{(j) T}\right]+\bar{H}_{k}^{(i)}\left(A_{k}, C_{k}\right) \mathcal{U}_{k}^{(j)}+D_{k}^{(i)} C_{k}^{T} \bar{H}_{k}^{(j) T}+R_{k}^{(i j)} .
$$

On the other hand, using again the expression of the observation predictor, we have that $E\left[\widehat{y}_{k / k-1}^{(i)} \mu_{k}^{(j) T}\right]=\bar{H}_{k}^{(i)}\left(A_{k}, C_{k}\right) \mathcal{U}_{k-1, k}^{(i j)}$ and expression (17) for $\Pi_{k}^{(i j)}$ is directly obtained. 


\section{Appendix C. Proof of Theorem 5.1}

By defining the matrices

$$
\begin{gathered}
K_{k, i}^{u} \equiv E\left[u_{k, i} u_{k, i}^{T}\right]=\sum_{j=1}^{i} \mathcal{U}_{k, j} \Pi_{k, j}^{-1} \mathcal{U}_{k, j}^{T}, \quad i=1, \ldots, m ; \quad K_{k, 0}^{u} \equiv 0, \\
\mathbf{K}_{k}^{\mathbf{U}} \equiv E\left[\mathbf{U}_{k} \mathbf{U}_{k}^{T}\right]=\sum_{h=1}^{k} K_{h, m}^{u}, \quad k \geq 1 ; \quad \mathbf{K}_{0}^{\mathbf{U}} \equiv 0,
\end{gathered}
$$

and applying (18)-(21), expressions (22)-(25)are deduced without trouble.

Also, taking into account (19) together with (22) for $\widehat{x}_{k, k, i-1}$, expression (26) for the innovation is clear.

To obtain expression (27) for $\Pi_{k, i}=E\left[\mu_{k, i} \mu_{k, i}^{T}\right]=E\left[y_{k}^{(i)} \mu_{k, i}^{T}\right]$, we use again that $y_{k}^{(i)}=\bar{H}_{k}^{(i)} x_{k}+\widetilde{H}_{k}^{(i)} x_{k}+v_{k}^{(i)}$ and, since $E\left[x_{k} \mu_{k, i}^{T}\right]=\mathcal{X}_{k, k, i}=\left(A_{k}, C_{k}\right) \mathcal{U}_{k, i}$, and $E\left[\left(\widetilde{H}_{k}^{(i)} x_{k}+v_{k}^{(i)}\right) \mu_{k, i}^{T}\right]=\mathcal{V}_{k, i}^{(i)}$, expression (27) is immediately deduced.

Finally, we prove expression (28) for

$$
\mathcal{V}_{k, j}^{(i)}=E\left[\left(\widetilde{H}_{k}^{(i)} x_{k}+v_{k}^{(i)}\right) y_{k}^{(j) T}\right]-E\left[\left(\widetilde{H}_{k}^{(i)} x_{k}+v_{k}^{(i)}\right) \widehat{y}_{k / k, j-1}^{(j) T}\right]
$$

The first expectation is given in (3) and, from (26), taking into account that $E\left[\left(\widetilde{H}_{k}^{(i)} x_{k}+v_{k}^{(i)}\right) \mathbf{U}_{k-1}^{T}\right]=0$, the second one satisfies:

$$
\begin{aligned}
& E\left[\left(\widetilde{H}_{k}^{(i)} x_{k}+v_{k}^{(i)}\right) \widehat{y}_{k / k, j-1}^{(j) T}\right] \\
& \quad=E\left[\left(\widetilde{H}_{k}^{(i)} x_{k}+v_{k}^{(i)}\right)\left(\bar{H}_{k}^{(j)}\left(A_{k}, C_{k}\right) u_{k, j-1}+\left(1-\delta_{j, 1}\right) \sum_{j^{\prime}=1}^{j-1} \mathcal{V}_{k, j^{\prime}}^{(i)} \Pi_{k, j^{\prime}}^{-1} \mu_{k, j^{\prime}}\right)^{T}\right] .
\end{aligned}
$$

Now, using (21) for $u_{k, j-1}$ and the definition of $\mathcal{V}_{k, j}^{(i)}$, we have

$$
E\left[\left(\widetilde{H}_{k}^{(i)} x_{k}+v_{k}^{(i)}\right) \widehat{y}_{k / k, j-1}^{(j) T}\right]=\left(1-\delta_{j, 1}\right) \sum_{j^{\prime}=1}^{j-1} \mathcal{V}_{k, j^{\prime}}^{(i)} \Pi_{k, j^{\prime}}^{-1}\left(\bar{H}_{k}^{(j)}\left(A_{k}, C_{k}\right) \mathcal{U}_{k, j^{\prime}}+\mathcal{V}_{k, j^{\prime}}^{(j)}\right)^{T}
$$

From (3) and the above expression, (28) is obtained and the theorem is proven.

\section{Appendix D. Proof of Theorem 5.2}

We start from the following identities satisfied by the centralized and sequential predictors and their error covariance matrices:

$$
\widehat{x}_{k / k-1}^{(C)}=\widehat{x}_{k / k-1}^{(S)}=\widehat{x}_{k / k-1} \quad \text { and } \quad \Sigma_{k / k-1}^{(C)}=\Sigma_{k / k-1}^{(S)}=\Sigma_{k / k-1} .
$$


From (7) and (8), taking into account that, from (29), $\widehat{x}_{k / k-1}^{(C)}=\left(A_{k}, C_{k}\right) u_{k-1}$ and that $\Sigma_{k / k-1}^{(C)}=A_{k} B_{k}^{T}-\left(A_{k}, C_{k}\right) K_{k-1}^{u}\left(A_{k}, C_{k}\right)^{T}$, we can write

$$
\begin{gathered}
\widehat{x}_{k / k}^{(C)}=\widehat{x}_{k / k-1}^{(C)}+\left(A_{k}, C_{k}\right) \mathcal{U}_{k} \Pi_{k}^{-1} \mu_{k}, \\
\Sigma_{k / k}^{(C)}=\Sigma_{k / k-1}^{(C)}-\left(A_{k}, C_{k}\right) \mathcal{U}_{k} \Pi_{k}^{-1} \mathcal{U}_{k}^{T}\left(A_{k}, C_{k}\right)^{T} .
\end{gathered}
$$

Analogously, from (22) and (23), we have that

$$
\begin{gathered}
\widehat{x}_{k / k}^{(S)}=\widehat{x}_{k / k-1}^{(S)}+\left(A_{k}, C_{k}\right) \sum_{j=1}^{m} \mathcal{U}_{k, j} \Pi_{k, j}^{-1} \mu_{k, j}, \\
\Sigma_{k / k}^{(S)}=\Sigma_{k / k-1}^{(S)}-\left(A_{k}, C_{k}\right) \sum_{j=1}^{m} \mathcal{U}_{k, j} \Pi_{k, j}^{-1} \mathcal{U}_{k, j}^{T}\left(A_{k}, C_{k}\right)^{T} .
\end{gathered}
$$

Hence, based on assumption (30), to prove that $\widehat{x}_{k / k}^{(C)}=\widehat{x}_{k / k}^{(S)}$ and $\Sigma_{k / k}^{(C)}=\Sigma_{k / k}^{(S)}$, we only need to prove that

$$
\left(A_{k}, C_{k}\right) \mathcal{U}_{k} \Pi_{k}^{-1} \mu_{k}=\left(A_{k}, C_{k}\right) \sum_{j=1}^{m} \mathcal{U}_{k, j} \Pi_{k, j}^{-1} \mu_{k, j}
$$

As in Lin and Sun (2019), the proof will be done by induction on the number of sensors, $m$. For $m=1$ it is immediately clear that the centralized and sequential filters are equal to each other. For $m=2$, the following notation is introduced for simplicity:

$$
\begin{aligned}
& \Delta_{k}^{(i)} \equiv \Sigma_{k / k-1} \bar{H}_{k}^{(i) T}+C_{k} D_{k}^{(i) T}, i=1,2, \\
& Y_{k}^{(i)}=y_{k}^{(i)}-\bar{H}_{k}^{(i)} \widehat{x}_{k / k-1}, i=1,2, \\
& \mathcal{R}_{k}^{(i, j)} \equiv E\left[\widetilde{H}_{k}^{(i)} A_{k} B_{k}^{T} \widetilde{H}_{k}^{(j) T}\right]+D_{k}^{(i)} C_{k}^{T} \bar{H}_{k}^{(j) T}+R_{k}^{(i j)}, i, j=1,2, \\
& \Phi_{k}^{(i, j)} \equiv \bar{H}_{k}^{(i)} \Delta_{k}^{(j)}+\mathcal{R}_{k}^{(i, j)}, i, j=1,2 .
\end{aligned}
$$

First, from the formulas of the centralized algorithm (Theorem 3.1) and using (30), we get

$$
\begin{aligned}
& \left(A_{k}, C_{k}\right) \mathcal{U}_{k}=\Sigma_{k / k-1} \bar{H}_{k}^{T}+C_{k} D_{k}^{T}, \\
& \Pi_{k}=\bar{H}_{k} \Sigma_{k / k-1} \bar{H}_{k}^{T}+H_{k} C_{k} D_{k}^{T}+E\left[\widetilde{H}_{k} A_{k} B_{k}^{T} \widetilde{H}_{k}^{T}\right]+D_{k} C_{k}^{T} \bar{H}_{k}^{T}+R_{k}, \\
& \mu_{k}=y_{k}-\bar{H}_{k} \widehat{x}_{k / k-1},
\end{aligned}
$$

Consequently, from (32), it is clear that

$$
\left(A_{k}, C_{k}\right) \mathcal{U}_{k} \Pi_{k}^{-1} \mu_{k}=\left(\begin{array}{ll}
\Delta_{k}^{(1)} & \Delta_{k}^{(2)}
\end{array}\right)\left(\begin{array}{cc}
\Phi_{k}^{(1,1)} & \Phi_{k}^{(1,2)} \\
\Phi_{k}^{(2,1)} & \Phi_{k}^{(2,2)}
\end{array}\right)^{-1}\left(\begin{array}{c}
Y_{k}^{(1)} \\
Y_{k}^{(2)}
\end{array}\right)
$$

Now, using that

$$
\left(\begin{array}{ll}
\Phi_{k}^{(1,1)} & \Phi_{k}^{(1,2)} \\
\Phi_{k}^{(2,1)} & \Phi_{k}^{(2,2)}
\end{array}\right)^{-1}=\left(\begin{array}{cc}
\Phi_{k}^{(1,1)-1}+\Phi_{k}^{(1,1)-1} \Phi_{k}^{(1,2)} \Theta_{k}^{-1} \Phi_{k}^{(2,1)} \Phi_{k}^{(1,1)-1} & -\Phi_{k}^{(1,1)-1} \Phi_{k}^{(1,2)} \Theta_{k}^{-1} \\
-\Theta_{k}^{-1} \Phi_{k}^{(2,1)} \Phi_{k}^{(1,1)-1} & \Theta_{k}^{-1}
\end{array}\right)
$$


where $\Theta_{k}=\Phi_{k}^{(2,2)}-\Phi_{k}^{(2,1)} \Phi_{k}^{(1,1)-1} \Phi_{k}^{(1,2)}$, the following expression for the left-hand side of (31) is obtained:

$$
\begin{aligned}
\left(A_{k}, C_{k}\right) \mathcal{U}_{k} \Pi_{k}^{-1} \mu_{k}= & \Delta_{k}^{(1)} \Phi_{k}^{(1,1)-1} Y_{k}^{(1)} \\
& +\left(\Delta_{k}^{(2)}-\Delta_{k}^{(1)} \Phi_{k}^{(1,1)-1} \Phi_{k}^{(1,2)}\right) \Theta_{k}^{-1}\left(Y_{k}^{(2)}-\Phi_{k}^{(2,1)} \Phi_{k}^{(1,1)-1} Y_{k}^{(1)}\right) .
\end{aligned}
$$

Next, from the formulas of the sequential algorithm (Theorem 5.1), using again (30) and (32), it is deduced that

$$
\begin{aligned}
& \left(A_{k}, C_{k}\right) \mathcal{U}_{k, 1}=\Delta_{k}^{(1)}, \quad\left(A_{k}, C_{k}\right) \mathcal{U}_{k, 2}=\Delta_{k}^{(2)}-\Delta_{k}^{(1)} \Phi_{k}^{(1,1)-1} \Phi_{k}^{(1,2)}, \\
& \Pi_{k, 1}=\Phi_{k}^{(1,1)}, \quad \Pi_{k, 2}=\Theta_{k}, \\
& \mu_{k, 1}=Y_{k}^{(1)}, \quad \mu_{k, 2}=Y_{k}^{(2)}-\Phi_{k}^{(2,1)} \Phi_{k}^{(1,1)-1} Y_{k}^{(1)}
\end{aligned}
$$

Hence, the right-hand side of (31) satisfies

$$
\begin{aligned}
\left(A_{k}, C_{k}\right) \sum_{j=1}^{2} \mathcal{U}_{k, j} \Pi_{k, j}^{-1} \mu_{k, j} & =\Delta_{k}^{(1)} \Phi_{k}^{(1,1)-1} Y_{k}^{(1)} \\
+ & \left(\Delta_{k}^{(2)}-\Delta_{k}^{(1)} \Phi_{k}^{(1,1)-1} \Phi_{k}^{(1,2)}\right) \Theta_{k}^{-1}\left(Y_{k}^{(2)}-\Phi_{k}^{(2,1)} \Phi_{k}^{(1,1)-1} Y_{k}^{(1)}\right) .
\end{aligned}
$$

From (33) and (34), expression (31) is proven for $m=2$. The rest of the proof is analogous to that of Theorem 2 in Lin and Sun (2019), so it is omitted. 